\title{
Combination of Reverse Shoulder Prosthesis, Elbow Prosthesis, and Polyetheretherketone: A Modified Total Humeral Replacement for Osteosarcoma Humerus Treatment
}

\author{
Dung Trung Tran ${ }^{a, b}$ Thanh Duc Tran ${ }^{a, b}$ Son Ba Nguyen ${ }^{c}$ \\ Sang Quang Tran Nguyen ${ }^{a, b}$ Quang Minh Dang ${ }^{a, b}$ Cong Van Tran ${ }^{b}$ \\ aDepartment of Orthopaedic Surgery, College of Health Science, VinUniversity, Hanoi, \\ Vietnam; ' Orthopaedic and Sports Medicine Center, Vinmec Healthcare System, Hanoi, \\ Vietnam; 'Department of General Surgery, Hanoi Medical University, Hanoi, Vietnam
}

\section{Keywords}

Osteosarcoma - Modified - total humerus replacement - Reverse shoulder prosthesis - Elbow prosthesis - Polyetheretherketone - Case report

\begin{abstract}
Total humeral replacement is a complex surgery that requires many challenges to overcome such as the weight of the implant material and the shoulder function due to extensive resection of the rotator cuff. Improvements in implants material that is lighter than usual can lead to higher surgery success rates. We present a patient who was diagnosed with osteosarcoma of the right humerus. The patient received 2 cycles of MAP chemotherapy (included: doxorubicin, cisplatin, and methotrexate) before surgery. He underwent radical resection of osteosarcoma and total humerus replacement with a modified total humeral material. The purpose of this improvement was to reduce the implant's weight and to improve postoperative recovery. Six months after the surgery, the weight-bearing ability of the patient's shoulder within a wide range of movement has restored; the shoulder, elbow, and hand can move in a controlled way. Despite the short postoperative follow-up time, the improvement in the modified technique has brought many positive results. Total humerus replacement, which combines the reverse shoulder prosthesis, elbow prosthesis, and polyetheretherketone, is an appropriate solution for patients with osteosarcoma of the humerus instead of custom-made humerus megaprosthesis.
\end{abstract}




\section{Introduction}

Osteosarcoma - primary malignant bone tumor - is a rare cancer with a worldwide incidence of 3.4 per million people per year [1]. One of the 3 most common sites of osteosarcoma is the humerus [2]. Treatment of humerus osteosarcoma is the same as the osteosarcoma on other sites which is mainly a combination of neoadjuvant chemotherapy and tumor wide resection [3]. Because of the development in diagnostic and management, the trend of limbsalvage surgery is increasingly popular. Though there is no significant difference in overall patient survival between limb-salvage surgery and amputation, this surgery is effective in preserving limb function and improving patients' psychological well-being [4].

Total humeral replacement is a relatively rare procedure. This treatment is often used when there is an extensive tumor; radical resection results in the insufficient remaining bone in both proximal and distal humerus for reconstruction; or the prior replacement surgery is failed [5]. Because the whole humerus containing the tumor is removed, this approach is more effective in eliminating radical cancer. The total humerus replacement with the modular replacement system (MRS) increases the success rate of the surgery, thus becoming the preferred procedure by surgeons [6].

However, the prosthesis reconstruction with the MRS from heavily weighted titanium alloy for teenage patients makes them difficult to recover during early-stage of rehabilitation [7]. Thus, reducing the weight of the prosthesis is a significant innovation and supports the rehabilitation phase.

A case of total humeral replacement for a bone cancer patient using a combination of titanium alloy prosthesis and polyetheretherketone (PEEK) was described in our report. This case report was made according to the SCARE 2020 guideline [8].

\section{Case Presentation}

A Vietnamese 19-year-old man presented to another hospital with a complaint of pain in the right humerus, and the biopsy revealed osteosarcoma. He had 2 cycles of preoperative chemotherapy, named MAP. After that, he came to our center with a desire to preserve his arm. There was no history of osteosarcoma within the family.

At the time of presentation, the patient was $165 \mathrm{~cm}$ tall and weighed $50 \mathrm{~kg}$. On examination, we found that the patient's upper arm was slightly swollen. He felt pain when he flexed his shoulders to $90^{\circ}$. On X-ray, we observed bone destruction, periosteal reaction, and a pathological fracture revealed on the proximal one-third of the humerus. On the computed tomography (CT) image, there was no sign of any cortical break in the one-third lower part (Fig. 1). However, on the magnetic resonance imaging image, an increased signal of tumor was extended $22 \mathrm{~cm}$ along the proximal to the distal part of the humerus (Fig. 2). The total length of the humerus was $27 \mathrm{~cm}$. So, the normal part of the remaining distal humerus was of insufficient length to perform reconstruction. The patient underwent the other assessment for bone cancers before being diagnosed with osteosarcoma of the right humerus stage IIB.

Based on the patient's most recent CT scan data, total humerus resection with tumor was indicated and planned for reconstruction. With the experience from the modular megaprosthesis total humerus replacement we performed before for another Vietnamese patient [7], we decided to replace the humerus with available reverse shoulder prosthesis and elbow prosthesis (Chunli Zhenda Medical Instruments Company, Beijing, China) and used a connection from PEEK material manufactured in Vietnam. This improvement will give 2 advantages in comparison with the previous one we performed: first, with the reverse shoulder prosthesis, shoulder elevation function will be better; second, the PEEK connection will reduce the weight

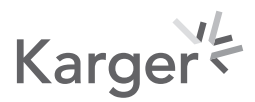




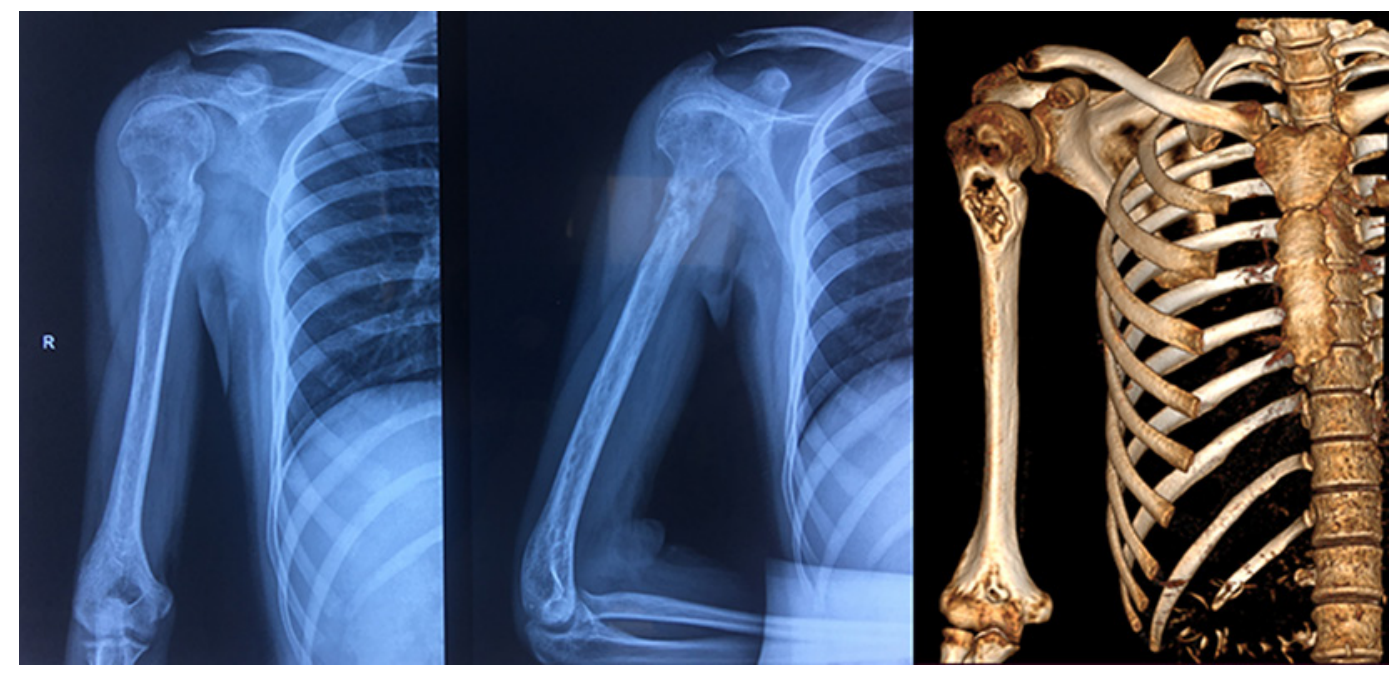

Fig. 1. X-ray and CT findings at presentation. CT, computed tomography.

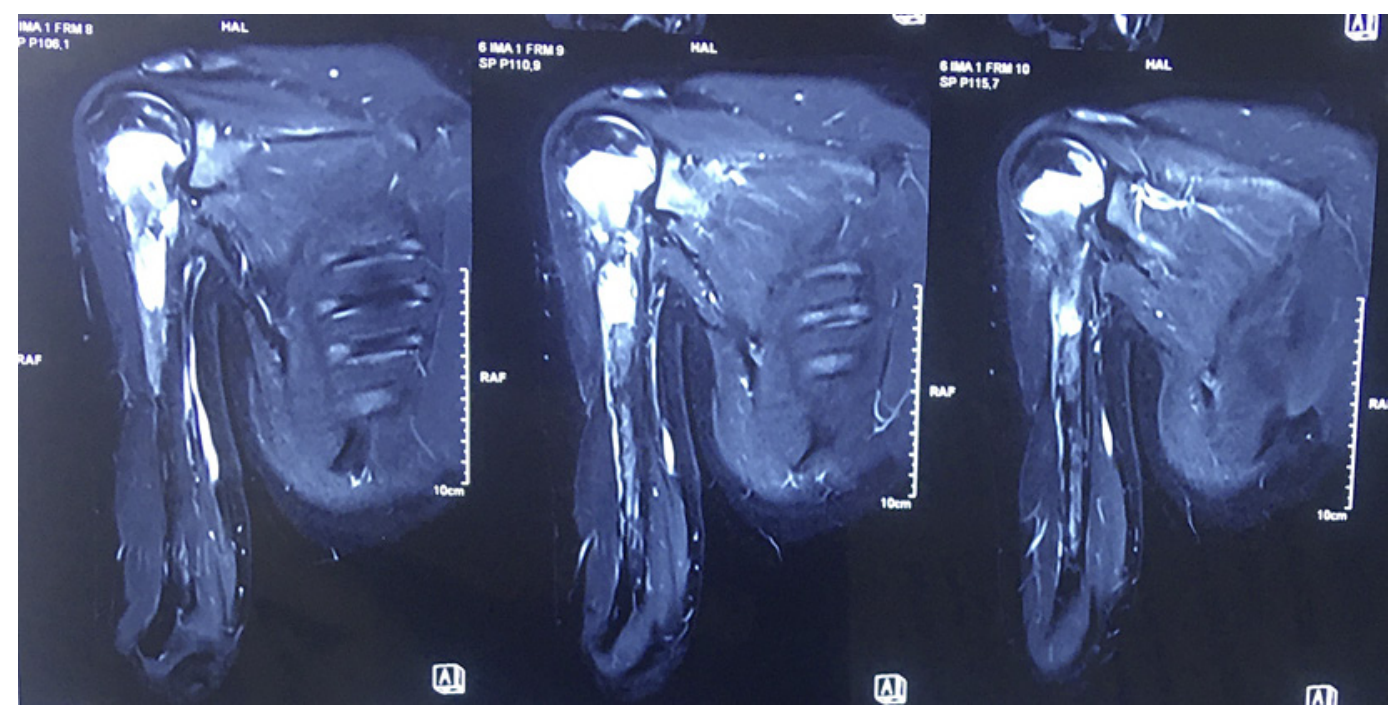

Fig. 2. Extended tumor along the proximal to the distal part of humerus on MRI. MRI, magnetic resonance imaging.

of combination. The patient was operated on by a team of surgeons with many experiences related to limb-salvage surgery in the treatment of bone tumors at a large orthopedic center in Vietnam.

He was placed in a supine position and was under endotracheal anesthesia during the procedure. The incision was made following the line of the deltopectoral groove proximal one-third of the humerus, then extended by anterolateral extension through the middle onethird part to complete the distal humerus and elbow joint approach. The attachment points of the pectoralis major, subscapularis, teres minor, teres major, latissimus dorsi, and biceps (long heads) were exposed, cut, and marked, respectively. Similarly, each part of the rotator cuff tendon and posterior articular capsule were also identified and tagged, and the humeral head was dislocated from the shoulder joint and rotator cuff tendon structures. In the next step, we released and marked the deltoid muscle from its insertion on the humerus ( $1 \mathrm{~cm}$ away). 
Fig. 3. Total humerus after resection.

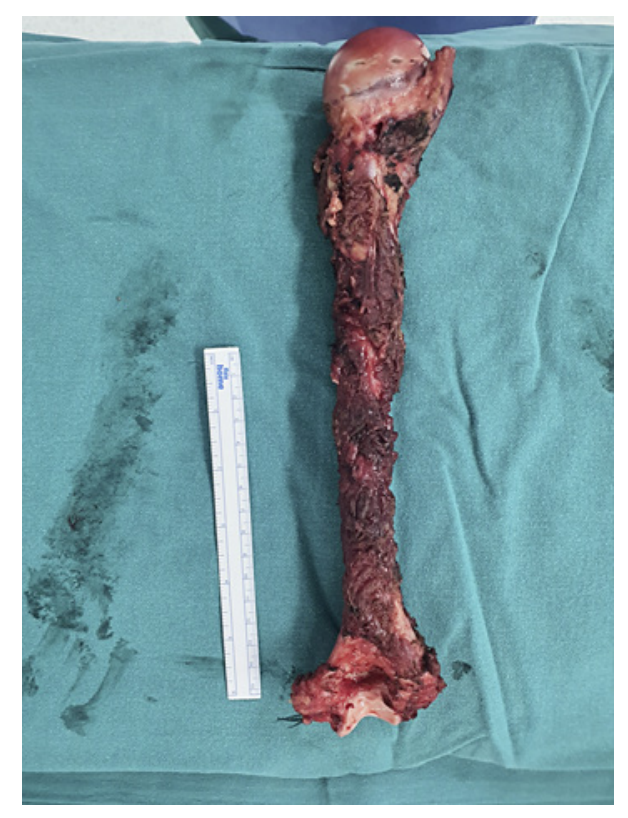

Fig. 4. Prostheses were linked together with bone cement into 1 unit.

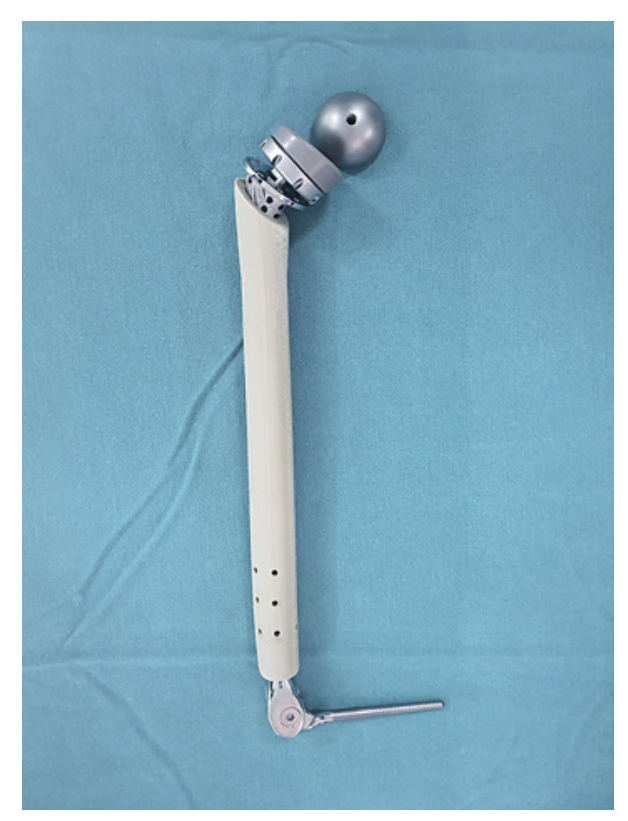

The radial nerve and the medial neurovascular structures (include: brachial artery, ulna nerve, and median nerve) were identified and preserved. In the distal one-third of the humerus, we dissected the middle of the brachial muscle, the radial nerve, and the ulnar were identified and protected, respectively. However, the dissection was quite complicated, thus causing damage to part of the ulnar nerve. Last, the whole humerus was released and removed (Fig. 3).

Our surgery included reverse shoulder arthroplasty and elbow arthroplasty with semiconstrained hinge prosthesis. We connected them by the prosthetic humeral stem made from PEEK material which was designed as same as the original humeral bone. Those prostheses were linked together with bone cement into 1 unit (Fig. 4). Maximum preservation of software tissue was carefully considered in our procedure. Based on preoperative data, the holes were 


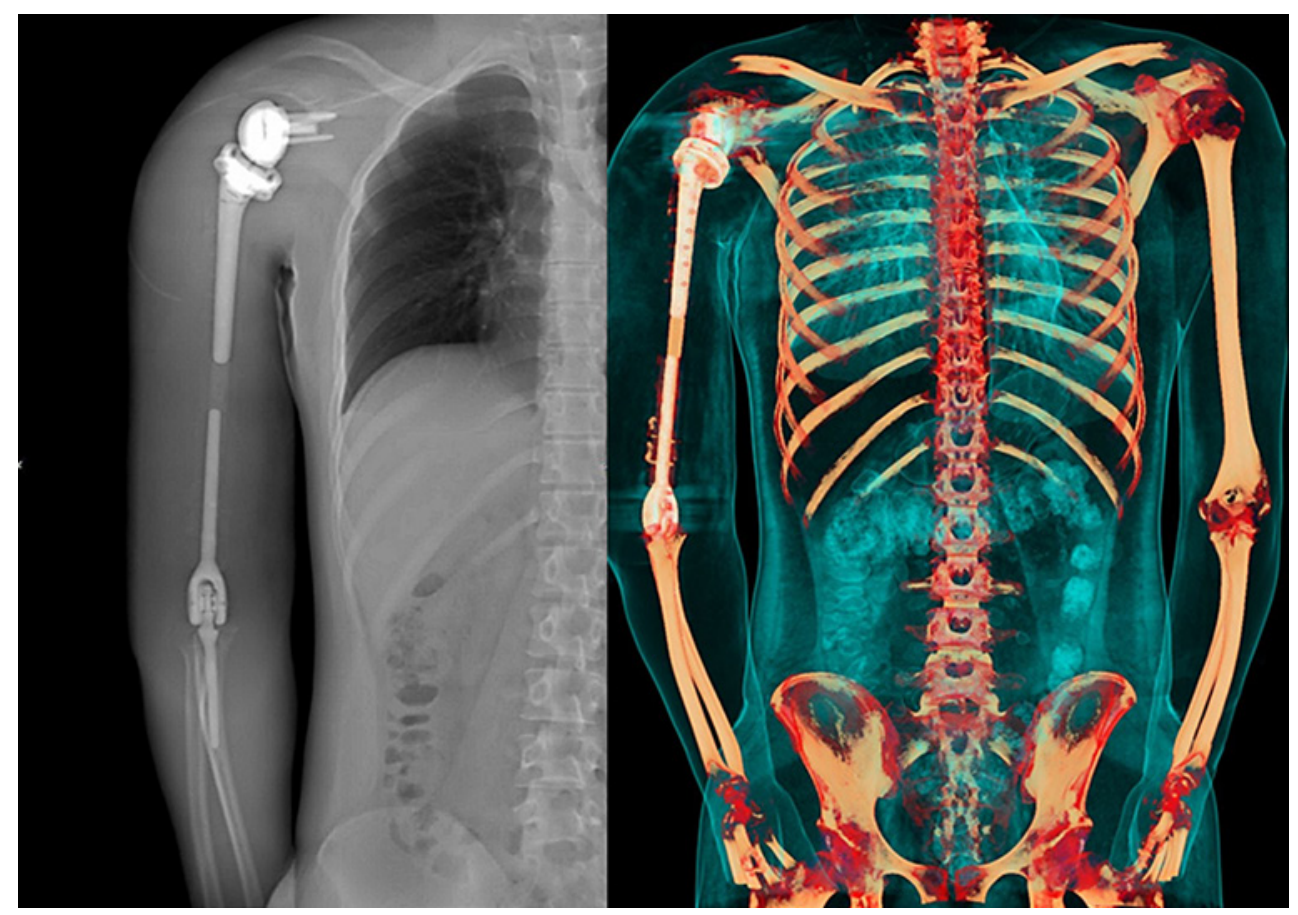

Fig. 5. X-ray and CT finding after surgery. CT, computed tomography.

predrilled on the PEEK segment at positions that are similar to the attachment points of the rotator cuff tendons, deltoid tendons, biceps, and attachment points of the lateral and medial epicondyle. When the total humeral prosthesis was well-placed, we fixed the attachment points of the abovementioned soft tissues to the corresponding pre-calculated positions using nonabsorbable suture threads.

When conducting re-examination after surgery by CT scan 3D rendering, the shoulder and elbow joints were observed to be in the correct positions. The modular prostheses comprise the reversed shoulder joint and PEEK segment, and the elbow joint was well assembled as preoperative planning (Fig. 5).

During clinical examination on the first day after the surgery, the patient was able to sit up, passively flexed his elbow, and moved the arm well. There was slight numbness in the dominant arm of the ulnar nerve. The patient was rehabilitated immediately. He was discharged 5 days after surgery and had regular outpatient rehabilitation thereafter. Postoperative histopathological examination showed a conventional osteosarcoma and fibroblastic variant with 80\% necrosis, which means HUVOS II after chemotherapy (Fig. 6).

After 6 months of postoperative observation, the patient has completed the chemotherapy regimen after surgery and has been able to perform daily activities without difficulties. Shoulder abduction was $75^{\circ}$ and flexion was $45^{\circ}$. The MSTS functional score of the patient was 26/30 which was excellent (Fig. 7).

\section{Discussion}

Total humeral replacement surgery in osteosarcoma patients has always presented many challenges to the surgeon, and there have been few reports in the literature [9-11]. The humerus, especially the proximal part, is 1 of the 3 most common sites for osteosarcoma [2]. 


\section{Case Reports in Oncology}

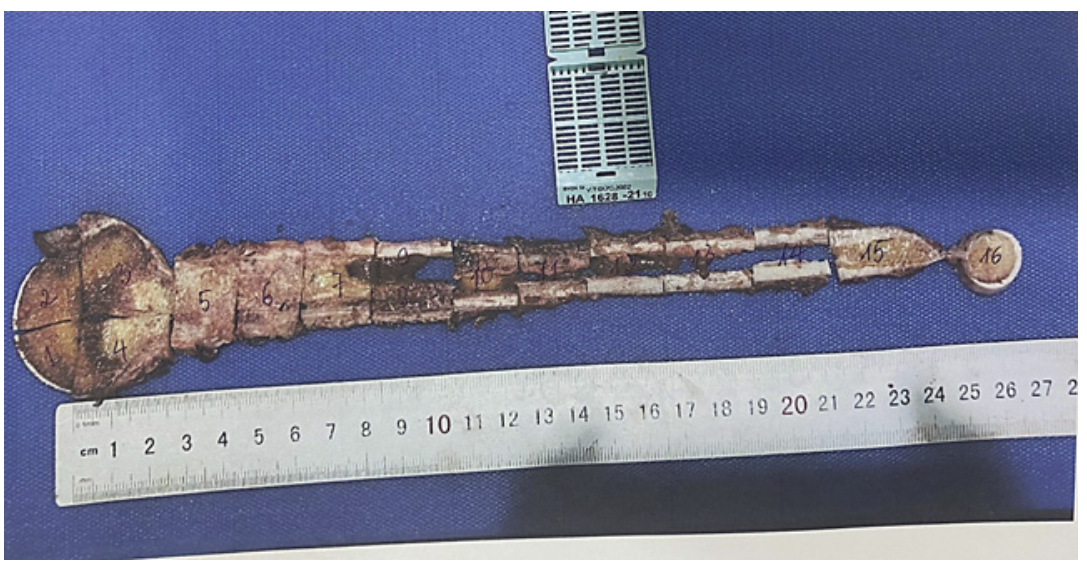

Fig. 6. Gross dissection map of the humerus.

Fig. 7. Patient shows his shoulder movement.

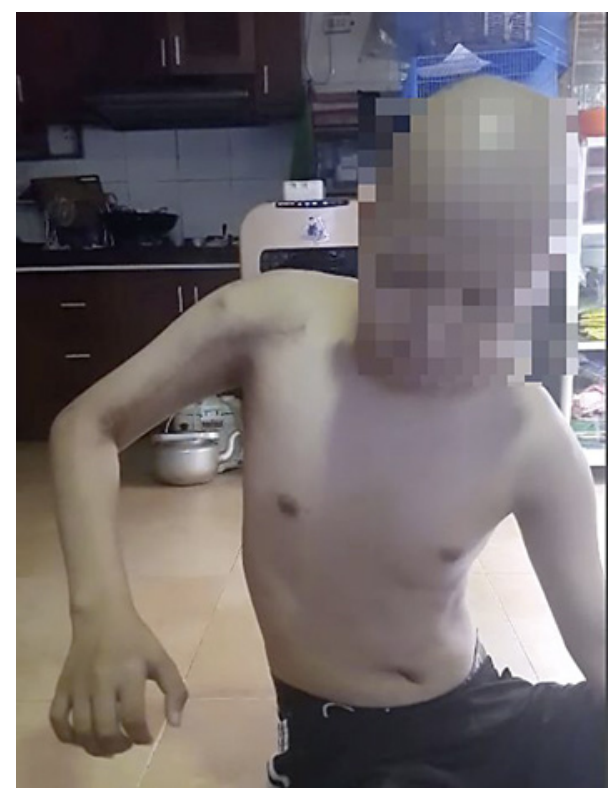

Our patient was a 19-year-old male who was diagnosed with primary humeral cancer. The patient underwent radical resection of the tumor and total humerus replacement because the tumor was extended along the proximal to the distal part of the humerus. Despite that limb-salvage surgery did not improve the survival rate of the patient compared to amputation, it helped to offer a better function of the limb and widely be accepted by patients and relatives [11,12].

Before the surgery, our patient completed the neoadjuvant chemotherapy regimen for 6 cycles with a good response in terms of clinical. Chemotherapy has been shown to increase the survival rate of patients after surgery by reducing recurrence and metastasis rates. In addition, chemotherapy also makes the surgery easier by reducing the size of the tumor and clarifying a better tumor margin demarcation [6].

We approached with the skin incision along the deltopectoral groove extending to the lateral side of the elbow. This approach is simpler than that of Prabowo and Reksoprodjo [6] as they had used a posterior curve to incorporate excision of the previous biopsy tract. Because the skin incision tends to run from anterior to lateral, when exposing the distal humerus, dissection of the ulnar nerve is difficult. We were very careful, but the ulnar nerve 
was partially damaged during the exposure. This problem did not leave clinical consequences. The movement of his hand after surgery was not affected; there was numbness in the fourth and fifth fingers but gradually improved after that. Kotwal et al. [4] undertook a large retrospective study of 20 patients and revealed the complication data as follows: 1 case of infection, 1 case of aseptic loosening, 3 cases of dislocation, and no case related to nerve damage.

This case report emphasizes the combination of locally available materials to produce a total humerus replacement for the patient. We used the reverse shoulder and conventional elbow joints which were suitable and available. Both prosthesis components were joined by a PEEK material in the form of an appropriate size tube that determined carefully the length during preoperative planning. This PEEK segment was made in Vietnam and was sterilized before the procedure. Three components were then assembled by bone cement into 1 unit. This kind of modified prostheses have been introduced as a "new modular prosthetic upper limb" when it is much lighter than the metal MRS popularly used in the world. We had experienced in 1 previous case of total humerus replacement with a teenage patient. In this case, the metal prosthesis MRS was used. The weight of the implant material affected the bearing ability of the patient's shoulder resulting in difficult and limited movement [7]. That is a reason this improvement is used to help to increase the ability to practice rehabilitation for patients after surgery. Additionally, the cost savings of this modified prosthetic made is widely accepted among patients and families, though this reason does not determine our professional decision. This is not a conventional treatment option for the total humerus replacement in our center but rather an option for the specific case.

To our knowledge, this is only the second reported case in the literature on the material improvement for the total humeral replacement after Prabowo's and Reksoprodjo publication [6]. In 2019, Prabowo reported a case of a 20 -year-old female patient who was diagnosed with humeral osteosarcoma. The technique of modified total humeral replacement was applied for this case. He applied a similar approach as our case with a combination of different materials to assemble them into the whole humerus. The difference between Prabowo and our case is that we used a part made of PEEK material with cement to attach the shoulder and elbow joint components. Therefore, the shape of the modified prosthesis was well-shaped immediately during intraoperative. In addition, reverse total shoulder arthroplasty helps patients improve shoulder function better than conventional replacement. Six months after the surgery, our patient had a relative range of motion with the shoulder abducted to $75^{\circ}$ and flexed to $45^{\circ}$. The patient in Prabowo's case report described excellent function with an MSTS score of $83 \%$. She was able to write comfortably after 2 months. The durability of the module recorded at the last follow-up after 3 years was stable without any looseness, infection, or fracture. Our biggest concern, in this case, is the longevity of the prostheses because 4 materials were assembled into 1 module during the implantation process. A long-term follow-up is needed to evaluate the durability when using such material combinations.

This case is the first modified total humeral replacement surgery performed in Vietnam. Our experience is quite limited, especially with this type of surgery. The limitation of this case report is the short follow-up time. Long-term follow-up needs to be conducted to evaluate the metastasis of tumor, recovery of patient, and longevity of our modified total humeral replacement module. With the immediate positive effects of this approach, we expect that this improvement of material will be an important step in improving the treatment of patients in the future.

\section{Conclusion}

Total humeral replacement surgery is a complex procedure among treatments for bone cancer. Modified material with the combination of reverse shoulder prosthesis, elbow prosthesis, and PEEK is an appropriate choice for patients with humeral tumors. This is a suitable treatment option for the patient and increases the functional efficiency of the humerus after surgery.

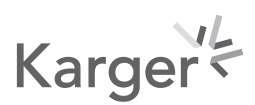




\section{Case Reports in Oncology}

\begin{tabular}{l|l}
\hline Case Rep Oncol 2022;15:12-20 \\
\hline DOI: 10.1159/000519599 & $\begin{array}{l}\text { ○ 2022 The Author(s). Published by S. Karger AG, Basel } \\
\text { www.karger.com/cro }\end{array}$ \\
\hline
\end{tabular}

Tran et al.: Combination of Material: A Modified Total Humeral Replacement

\section{Statement of Ethics}

The procedures used in this study adhere to the tenets of the Declarations of Helsinki. Case reports are approved by the Joint Consultation for Approval of Surgery and are granted an exemption from requiring ethical approval at the Vinmec Healthcare System. Written informed consent was obtained from the patient for publication of this case report and any accompanying images. A copy of the written consent is available for review by the Editor-in-Chief of this journal.

\section{Conflict of Interest Statement}

The authors have no conflicts of interest to declare.

\section{Funding Sources}

The authors declare that they have no known competing financial interests or personal relationships that could have appeared to influence the work reported in this paper.

\section{Author Contributions}

Dung Trung Tran, the main doctor, conceived the original idea manuscript and operated the patient. Thanh Duc Tran summed up, operated the patient, and revised the manuscript. Son Ba Nguyen and Quang Minh Dang operated the patient and wrote the manuscript. Sang Quang Tran Nguyen operated the patient and revised the manuscript. Cong Van Tran wrote the manuscript. All the authors read and approved the final manuscript.

\section{Data Availability Statement}

All relevant data are provided in the manuscript. Further inquiries can be directed to the corresponding author.

\section{References}

1 Mirabello L, Troisi RJ, Savage SA. International osteosarcoma incidence patterns in children and adolescents, middle ages and elderly persons. Int J Cancer. 2009;125(1):229-34.

2 Ritter J, Bielack SS. Osteosarcoma. Ann Oncol. 2010;21:vii320-5.

3 Böhler C, Brönimann S, Kaider A, Puchner SE, Sigmund IK, Windhager R, et al. Surgical and functional outcome after endoprosthetic reconstruction in patients with osteosarcoma of the humerus. Sci Rep. 2018;8:16148.

4 Kotwal S, Moon B, Lin P, Satcher R, Lewis V. Total humeral endoprosthetic replacement following excision of malignant bone tumors. Sarcoma. 2016;2016:6318060.

5 Bernthal NM, Upfill-Brown A, Burke ZDC, Greig D, Hwang R, Crawford B, et al. Long term outcomes of total humeral replacement for oncological reconstructions: a single institution experience. J Surg Oncol. 2020; 122(4):778-86.

6 Prabowo Y, Reksoprodjo AY. Modified total humeral replacement on unusual osteosarcoma of the humerus: a case report. Int J Surg Case Rep. 2019;58:132-7.

7 Trung DT, Huu MN, Khanh TL, Minh TN, Quan SNT, Duc TT. Total humerus replacement for Ewing sarcoma in adolescent patient: a case report in Vietnam. J Orthop Trauma Surg Relat Res. 2020;15(2).

8 Agha RA, Franchi T, Sohrabi C, Mathew G, Kerwan A, SCARE Group. The SCARE 2020 Guideline: updating consensus surgical case report (SCARE) Guidelines. Int J Surg. 2020;84:226-30. 
9 Natarajan M, Sameer M, Kunal D, Balasubramanian N. Custom-made endoprosthetic total humerus reconstruction for musculoskeletal tumours. Int Orthop. 2012;36(1):125-9.

10 Funovics PT, Schuh R, Adams SB, Sabeti-Aschraf M, Dominkus M, Kotz RI. Modular prosthetic reconstruction of major bone defects of the distal end of the humerus. J Bone Joint Surg Am. 2011;93(11):1064-74.

11 Kotwal S, Moon B, Lin P, Satcher R, Lewis V. Total humeral endoprosthetic replacement following excision of malignant bone tumors. Sarcoma. 2016;2016:6318060.

12 Rougraff BT, Simon MA, Kneisl JS, Greenberg DB, Mankin HJ. Limb salvage compared with amputation for osteosarcoma of the distal end of the femur. A long-term oncological, functional, and quality-of-life study. J Bone Joint Surg Am. 1994;76(5):649-56. 